\title{
Die Casting Plunger Pressing Velocity and Analysis of Its Influence on a Permanent Deformation Value of a Casting Made from an ENAC 47100 Alloy
}

Assoc. Prof. Paško Ján, MSc., PhD., Department of Technical Devices Design, Technical University of Košice, Faculty of Manufacturing Technologies with seat in Prešov, Slovak Republic

A die casting plunger velocity inside a filling chamber of a pressure die casting machine is one of the most significant factors of a pressure die casting. This velocity determines the regime of a die cavity filling, thus influencing both inner and surface quality of castings. A selection of a correct velocity of a die cavity filling depends on such factors as an alloy type, a casting complexity, a ratio of a slot area to a casting area, etc. A static pressure test can be used to determine a permanent deformation value by which a casting quality can be considered.

Keywords: die casting plunger pressing velocity, ENAC 47100 alloy, permanent deformation, homogeneity of a casting

\section{References}

[1] GME 60 007, Aluminiumteile fur Treibwerklagerung und Aluminiuminnenkerne von Dämpfungsblocken, Conti Tech Vegum VC s.r.o.Úsek riadenia kvality, 25.5.2001.

[2] GME 60 156, Test Method for Determining the Compression Properties of Centre Tubes, Vauxhallm Morors LTD, 11.1.2001.

[3] MALIK, J.: Technológia tlakového liatia hliníka, Habilitačná práca, TU-HF Košice, 2008.

[4] RAGAN, E a kol.: Liatie kovov pod tlakom, FVT Prešov, 2007.

[5] STN EN 1706, Hliník a zliatiny hliníka, Odliatky, Chemické zloženie a mechanické vlastnosti.

[6] NOVÁ, I., SOLFRONK, P., NOVÁKOVÁ, I. Vliv tlaku na strukturu slitiny hliníku AlCu4MgTi. In Strojírenská technologie, 2010, roč. XV, č. 3,. Str. $11-17$, ISSN 1211-4162.

[7] MICHNA, Š. Legování hliníkových slitin pomocí chromových legovacích tablet. In Strojírenská technologie, 2010, roč. XV, č.1,. Str. $22-26$, ISSN 1211-4162.

Reviews:

Prof. Iva Nová, MSc., Ph.D. Assoc. Prof. Dana Bolibruchová, MSc., PhD. 